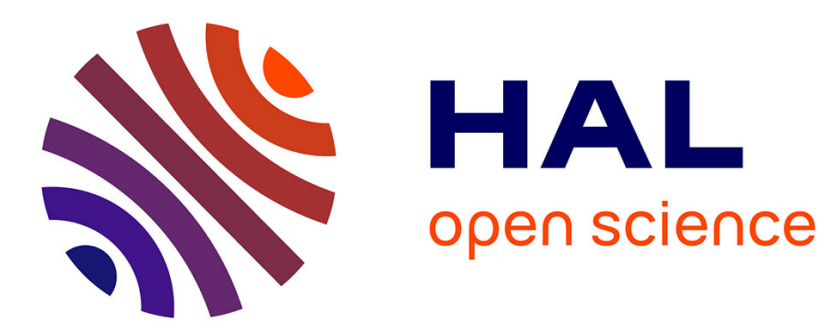

\title{
Design and Evaluation of a Wideband Full-Duplex OFDM System Based on AASIC
}

\author{
Zhaowu Zhan, Guillaume Villemaud, Jean-Marie Gorce
}

\section{To cite this version:}

Zhaowu Zhan, Guillaume Villemaud, Jean-Marie Gorce. Design and Evaluation of a Wideband FullDuplex OFDM System Based on AASIC. IEEE Personal Indoor and Mobile Radio Communications (PIMRC), 2013, Sep 2013, London, United Kingdom. pp.68 - 72, 10.1109/PIMRC.2013.6666106 . hal-00920918

\section{HAL Id: hal-00920918 https://hal.inria.fr/hal-00920918}

Submitted on 19 Dec 2013

HAL is a multi-disciplinary open access archive for the deposit and dissemination of scientific research documents, whether they are published or not. The documents may come from teaching and research institutions in France or abroad, or from public or private research centers.
L'archive ouverte pluridisciplinaire HAL, est destinée au dépôt et à la diffusion de documents scientifiques de niveau recherche, publiés ou non, émanant des établissements d'enseignement et de recherche français ou étrangers, des laboratoires publics ou privés. 


\title{
Design and Evaluation of a Wideband Full-Duplex OFDM System Based on AASIC
}

\author{
Zhaowu Zhan, Guillaume Villemaud and Jean-Marie Gorce \\ Université de Lyon, INRIA \\ INSA-Lyon, CITI-INRIA, F-69621, Villeurbanne, France \\ Email: zhaowu.zhan@insa-lyon.fr
}

\begin{abstract}
Existing methods of self-interference cancellation have greatly reduced the strong self-interference signal for the practical design of Full-Duplex (FuDu) wireless systems. However, current FuDu wireless is hard to be put into practice due to the reason that residual self-interference after interference cancellation is still much stronger than the thermal noise. In this paper, a wideband FuDu OFDM system with a single path self-interference channel is developed based on active analog self-interference cancellation (AASIC) at the RF component. Furthermore, a mathematical model of the wideband FuDu OFDM wireless system and the AASIC at the RF component are presented and elaborated. The performance of this FuDu system is evaluated by ADS-Matlab co-simulation based on the IEEE 802.11g system parameters. The comparison of the spectrum power of signals before and after the AASIC and the bite error rate (BER) performance of the FuDu OFDM wireless system show that the strong self-interference can be significantly reduced to almost noise level.
\end{abstract}

Keywords-Full-Duplex; OFDM; Self-interference; Radio

\section{INTRODUCTION}

In recent years, there have been great progresses in digital signal processing and digital radio implementation. These provide the opportunity to push the concept of full-duplex $(\mathrm{FuDu})$ into reality. Current communication standards always share the medium with half-duplex principles: transmission and reception of signals are done in two separated time slots or two different frequency channels. The FuDu paradigm supposes that transceivers are able to transmit and receive at the same time on the same frequency band. Therefore, in $\mathrm{FuDu}$ wireless communications, a radio node not only receives the signal of interest from the distant node but also receives the signal from its own transmitter, which we call self-interference signal. Due to the distance path loss, the selfinterference signal is much stronger than the signal of interest, e.g. $60 \sim 100 d B$ in typical WLAN environment. If no measures are taken to this strong self-interference, the useful signal can be completely masked by the interfering signal at the receiver side. Thus, the key point for implementing a FuDu radio is to cancel the self-interference as much as possible, ideally to the noise level.

In fact, for wireless engineers, the strong self-interference made unthinkable implementation of a real FuDu system until research articles [1, 3-4]. The authors combine the methods of passive and active self-interference cancelation together to reduce the strength of self-interference to tolerable level, which enlightens researchers that FuDu wireless is actually feasible.
The passive method [5] [6]is based on antenna decoupling, spatially or with cross-polarization to reduce the power of the self-interference signal arrived at the receive antenna. The active methods $[1-3,5]$ take profit of the knowledge of the transmit information and estimation of the coefficients of the over the air self-interference channel to generate a copy of the self-interference signal, and then subtract this copy in the receiver chain. The active methods include active analog self-interference cancellation (AASIC) and active digital selfinterference cancellation (ADSIC). AASIC carry out the selfinterference subtraction in the analog domain before the received signal is digitized, while ADSIC subtracts the selfinterference in the digital domain after the ADC (Analog-todigital converter).

Up to now, the practical design of the FuDu wireless $[3,7]$ has made it possible to implement a real $\mathrm{FuDu}$ radio by using a combination of passive and active techniques. These research works have shown how much self-interference cancellation can be achieved by using different cancellation methods. Another practical design $[2,5]$ has formulated the experiment-based characterizations of a FuDu wireless system. The authors demonstrated that the amount of total active self-interference cancellation (ASIC) decreased if the amount of passive self-interference cancellation (PSIC) increased, while the total amount of self-interference cancellation increased if the amount of PSIC increased. They also found that the total amount of active suppression is not the linear addition of the amount of AASIC and the amount of ADSIC achieved individually. The total amount of ASIC is limited by the selfinterference power received. This means the more AASIC FuDu system obtains, the less ADSIC it obtains, and vice versa. The largest amount of ASIC can be obtained only when the exact self-interference channel coefficients are obtained. Obviously, it is not necessary to cascade an ADSIC following the AASIC if the self-interference is completely eliminated in the analog part. Thus the ADSIC is optional, because it can only cancel the residual self-interference related with the selfinterference signal after passive suppression and active analog suppression.

In this paper, a single channel $20 \mathrm{MHz}$ FuDu OFDM wireless system based on the method of AASIC at the RF front-end is developed and evaluated. The self-interference channel between the transmit antenna and receive antenna is assumed to be line of sight with one single dominant path. This assumption relies on the fact that the strength of the line of sight path will dominate the power of self-interference channel when the distance between transmit antenna and 
receive antenna at the FuDu radio node is of few centimeters while reflected paths should be in meters, therefore considered as negligible in this first study. For this single path selfinterference channel, relative simple AASIC can significantly reduce the self-interference to almost noise level for the design of FuDu wireless. While with respect to the multipath selfinterference channel, much more taps are needed to emulate the self-interference channel to craft the cancelation signal [6]. The BER performance of the FuDu system shows that it requires $3 \sim 4 d B$ more power of signal of interest to compensate the increased noise, if residual self-interference signal is regarded as thermal noise, to get the same BER performance as the wireless link without self-interference.

The rest of this paper is organized as follows. Section II presents the architecture of FuDu wireless, including basic principle of $\mathrm{FuDu}$, channel model, self-interference channel estimation, method of AASIC at the RF front-end and design of $\mathrm{FuDu}$ OFDM radio node. Then, the ADS-Matlab cosimulation results of this wideband FuDu OFDM wireless are shown in Section III. Finally, conclusion is drawn in Section IV.

\section{ARChitecture OF FULl-DUPLEX WirElesS}

In half-duplex wireless system, the transceiver uses orthogonal radio resource for transmitting and receiving. While in $\mathrm{FuDu}$ wireless system, the transceiver transmits and receives simultaneously on the same frequency. Figure 1 . shows the block diagram of FuDu architecture. The transmitted signal not only goes through the multipath fading channel and arrives at the receive antenna of the distant node but also experiences the line of sight wireless channel and arrives at the receive antenna of the local node. Therefore the signal received of each of these two nodes is composed of the signal of interest, the strong self-interference and the thermal noise. At the receiver side, the total received signal is scaled by the automatic gain controller (AGC) to the full dynamic range of $\mathrm{ADC}$, and then it is digitized and quantized by ADC. The stronger the self-interference is, the fewer useful information is included in digital samples, because the number of resolution bits of ADC is limited. In order to reduce this strong self-interference signal for implementing a FuDu radio, AASIC at the RF component is required.

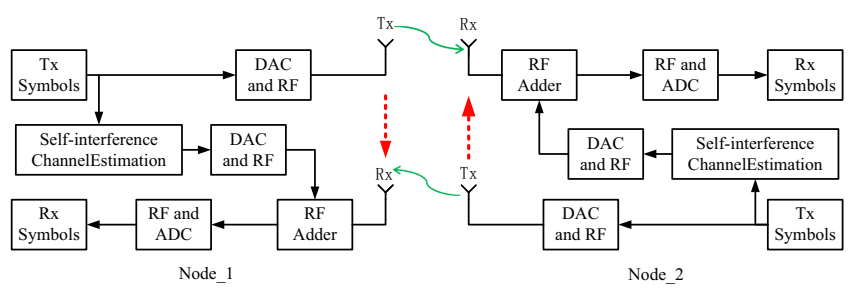

Fig. 1. Block Diagram of Full-Duplex wireless

\section{A. Channel Model}

In the indoor environment, the wireless channel between nodes can be modeled as typical indoor WLAN channel. Here, the indoor WLAN channel is assumed to be quasi-static. In other words, the channel condition is considered to be static during each transmit frame. The channel impulse response $h(t)$ can be expressed as:

$$
h(t)=\sum_{i=0}^{r-1} h_{i} \delta\left(t-\tau_{i}\right)
$$

where $r$ is total number of propagation paths, $h_{i}$ is the complex impulse response of the $i^{t h}$ path, $t$ is delay spread index and $\tau_{i}$ is the $i^{t h}$ path delay.

The characterizations of the self-interference channel are quite different from that of the multipath fading channel. As a consequence of proximity between transmit antenna and receive antenna, the power of line of sight path dominates the power of self-interference channel. Therefore the selfinterference channel is assumed to be a single delay path and the channel impulse response can be represented by:

$$
h(t)=h_{s i} \delta\left(t-\tau_{s i}\right)
$$

where $h_{s i}$ is the complex impulse response of the selfinterference channel and $\tau_{s i}$ is the delay of the selfinterference channel.

\section{B. Channel Estimation}

Typical channel estimation is either performed in the time domain to measure the channel impulse response or carried out in the frequency domain for estimating the transfer function in the specific frequency. In order to construct the cancellation signal in each subcarrier channel, the channel estimation is carried out in the frequency domain here. In the IEEE $802.11 \mathrm{~g}$ frames, the preamble consists of 10 identical short OFDM symbols each of length 16 and 2 identical long OFDM symbols each of length 64. Each long OFDM symbols is generated via IFFT of 52 known BPSK symbols and 12 nulls. The estimation of the coefficients of 52 subcarrier channels is carried out via dividing the received long OFDM symbols by the 52 known symbols. Then the AASIC at the RF component is based on the estimation of coefficient of each subcarrier channel. Even small error of subcarrier channel estimation may cause large residual self-interference. In order to avoid the channel estimation errors induced by residual self-interference, the long training symbols are sent from each transmitter radio on the orthogonal time slots.

\section{Active Analog Self-interference Cancelation}

In the IEEE 802.11g, Orthogonal Frequency Division Multiplexing (OFDM) has been selected as the key physical techniques for high speed transmission. The $20 \mathrm{MHz}$ system bandwidth is divided by OFDM into 64 orthogonal subcarrier channels, 48 of which are used for carrying data symbols and 4 of which are employed to transmit pilot symbols. Thus the wideband channel is divided into a number of narrowband frequency flat fading channels. This provides us the opportunity to generate the cancellation signal in the frequency domain by multiplying symbols to be transmitted with the estimated coefficients of subcarrier channels. And then the signal received is subtracted by the cancellation signal at the RF component to cancel the self-interference signal before it is down converted to baseband. 


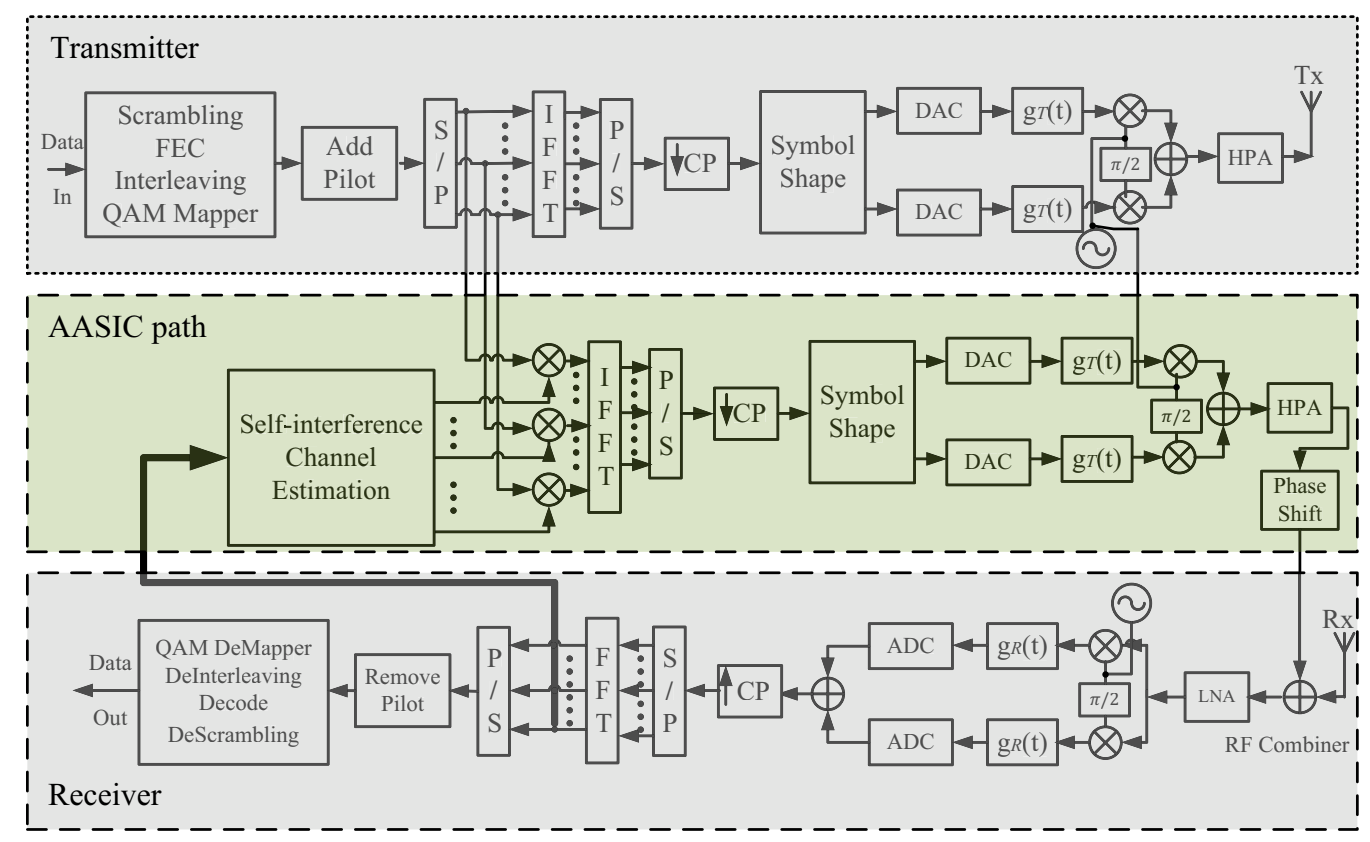

Fig. 2. Architecture of Full-Duplex OFDM radio node

In this paper, the transceiver is assumed to be ideal, i.e. the dynamic range of DAC/ADC is large enough, phase noise (PN) does not exist in the local oscillator and there is no I/Q imbalance. Transmit continuous baseband signal can be formulated by cascading time domain OFDM symbols one by one. By up-converting this baseband signal, RF signal which is to be emitted from the transmit antenna is generated. In practice, the OFDM signal is generated by using inverse DFT to modulate the transmit symbols. Let $X_{s}[k]$ denote the symbol carried by the $k^{t h}$ subcarrier in the $s^{t h}$ OFDM symbol. In order to facilitate the mathematic expression of OFDM signal, we limit one OFDM symbol in time domain to a time interval $T_{u}$. The continuous time domain signal $x_{s}(t)$ is therefore expressed as:

$$
x_{s}(t)=\frac{1}{T_{u}} \sum_{k=-\frac{N}{2}}^{\frac{N}{2}-1} X_{s}[k] e^{j 2 \pi \Delta f k t},(s-1) T_{u} \leq t<s T_{u}
$$

where $N$ is the total number of the subcarriers and $\Delta f$ denotes the subcarrier space. The signal is finally up converted to radio frequency:

$$
T_{x, s}(t)=\operatorname{Real}\left(x_{s}(t) e^{j 2 \pi f_{c} t}\right),(s-1) T_{u} \leq t<s T_{u}
$$

where $T_{x, s}(t)$ denotes the transmit RF signal and $f_{c}$ represents the carrier frequency. For simplifying the expression, $(s-$ 1) $T_{u} \leq t<s T_{u}$ wil be drapped in the following formulas. The emitted RF signal experiences multipath channel fading and then arrives at the receive antenna of a distance node. The received useful signal $R_{x, s}(t)$ is presented as:

$$
R_{x, s}(t)=\operatorname{Real}\left(\left(x_{s}(t) * h(t)\right) e^{j 2 \pi f_{c} t}\right)+n(t)
$$

where $*$ denotes convolution and $n(t)$ is the the AWGN noise which follows the distribution of $N\left(0, \delta_{n}{ }^{2}\right)$.
If we use $T_{x, s, s i}(t)=\operatorname{Real}\left(x_{s, s i}(t) e^{j 2 \pi f_{c} t}\right)$, where $x_{s, s i}(t)$ represents the continuous time domain selfinterference signal in the $s^{t h}$ OFDM symbol, to denote the transmit RF self-interference signal, the RF self-interference signal received at the receive antenna can be therefore written as:

$$
R_{x, s, s i}(t)=\operatorname{Real}\left(\left(x_{s, s i}(t) * h_{s i}(t)\right) e^{j 2 \pi f_{c} t}\right)
$$

Therefore the received signal at the receive antenna can be represented by:

$$
R_{s}(t)=R_{x, s}(t)+R_{x, s, s i}(t)
$$

The cancellation signal can be crafted by multiplying the coefficients of the self-interference subcarrier channel with the modulated symbols in the frequency domain. The coefficient of the self-interference channel is obtained from the channel estimation at the receiver side. Let the vector $\widetilde{H}_{s, s i}=\left[\widetilde{H}_{s, s i}[1], \widetilde{H}_{s, s i}[2],, \widetilde{H}_{s, s i}[N]\right]$ denotes $N$ coefficients of the estimated subcarrier channel at the $s^{\text {th }}$ time-interval in frequency domain, the baseband continuous time domain cancellation signal $x_{s, c}(t)$ is therefore expressed as:

$$
x_{s, c}(t)=\frac{1}{T_{u}} \sum_{k=-\frac{N}{2}}^{\frac{N}{2}-1} X_{s, s i}[k] \widetilde{H}_{s, s i}[k] e^{j 2 \pi \Delta f k t}
$$

where $X_{s, s i}[k]$ denotes the modulated symbol stransmitted from the local node. In order to make the cancellation signal match with the self-interference signal at the receiver side, we up-convert the baseband signal $x_{s, c}(t)$ to RF cancellation signal $C_{s}(t)$, which is expressed as:

$$
C_{s}(t)=\operatorname{Real}\left(x_{s, c}(t) e^{j 2 \pi f_{c} t}\right)
$$

The inverse version of the cancellation signal can be obtained by using $180^{\circ}$ phase shifter. 
Self-interference subtraction is implemented at the RF component by using RF combiner before the RF signal is down converted to baseband. Then, the residual received signal after AASIC is expressed as:

$$
R_{\text {residual }, s}(t)=R_{x, s}(t)+R_{x, s, s i}(t)-C_{s}(t)
$$

The residual self-interference after AASIC can be represented as:

$$
R_{\text {residual }, s}(t)=R_{x, s, s i}(t)-C_{s}(t)
$$

This component can be forced to zero when the exact coefficients are obtained in ideal case. However, it is difficult to get the exact coefficients of the self-interference channel because the channel estimation is at least influenced by the thermal noise $n(t)$, while we can reduce the self-interference which component is closely related to channel estimation in each subcarrier channel to almost noise [8].

\section{Full-Duplex OFDM Radio Node Design}

In order to decode the useful signal correctly, FuDu radio node must have the ability to suppress the interference signal from its own transmitter at the receiver chain. Fig 2. shows the architecture of a wideband FuDu OFDM radio node based on the physical layer of IEEE802.11g. The AASIC path is added to the FuDu OFDM radio in order to generate the cancellation signal for the implementation of AASIC at the RF component.

In our developed FuDu OFDM radio design, the additional hardware components required for AASIC at the RF component consist of one RF combiner, one $180^{\circ}$ phase shifter, two DACs and one RF frontend.

In the cancellation path, the component of selfinterference channel estimation is used for obtaining coefficient estimation of each subcarrier channel. Further, $\widetilde{H}_{s, s i}[k]$ denotes the magnitude and phase of the $k^{t h}$ subcarrier channel. The baseband cancellation signal $x_{s, c}(t)=\frac{1}{T_{u}} \sum_{k=-\frac{N}{2}}^{\frac{N}{2}-1} X_{s, s i}[k] \widetilde{H}_{s, s i}[k] e^{j 2 \pi \Delta f k t}$ is generated by OFDM modulating the new complex symbols sequence $X_{s, s i}[k] \widetilde{H}_{s, s i}[k], k \in[1, N]$. Then, another RF front-end is employed to up-convert this baseband cancellation signal to RF cancellation signal. After the HPA amplifying the RF cancellation signal to match the power of self-interference signal, $180^{\circ}$ phase shifter is used to get the inverse version. Then, the self-interference cancellation is carried out at the receiver chain by combining the signal received and the inverse version of cancellation signal via RF combiner.

In order to avoid the impact of phase noise in the local oscillator on the self-interference cancellation [8], the transmitter and the cancellation path use the same local oscillator. Furthermore, the AASIC is implemented by using RF combiner which combines the received signal and RF cancellation signal between the receiver antenna and low noise amplifier (LNA) to eliminate the influence of dirty RF at the receiver chain on the self-interference cancellation. After AASIC, the signal received including signal of interest, residual selfinterference and thermal noise will be further processed by the receiver chain performing the inverse operation as the transmitter chain.

\section{Simulation Results}

The simulation parameters of FuDu OFDM system are chosen as Table I. according to the IEEE $802.11 \mathrm{~g}$ standards.

TABLE I. SYSTEM PARAMETERS

\begin{tabular}{cc}
\hline \hline Parameter & Value \\
\hline Bandwidth & $20 \mathrm{MHz}$ \\
Total number of subcarriers & 52 \\
Number of data subcarrers & 48 \\
Number of pilot subcarriers & 4 \\
Bit rate & $36 \mathrm{Mbps}$ \\
Carrier frequency & $2.4 \mathrm{GHz}$ \\
IFFT/FFT period & $3.2 \mathrm{us}$ \\
GI duration & $0.8 \mathrm{us}$ \\
\hline
\end{tabular}

The system is developed on the system-level simulator ADS (Advanced Design System, Agilent) which permits to model a complete system from device to architecture. Matlab is used in co-simulation to process part of digital signal processing. In the FuDu OFDM system design, the assumption that $\mathrm{RF}$ front-ends are ideal, i.e. there is no phase noise in the local oscillator, no I/Q imbalance in RF modulation, no frequency offset and no non-linearity of HPA, is made.

During the ADS-Matlab co-simulation of the wideband FuDu OFDM wireless system, the power spectrum density of thermal noise is fixed to $-167 \mathrm{dBm} / \mathrm{Hz}$ aim to facilitate the power set up of signal of interest and self-interference signal. More specifically, the power of self-interference signal can be easily calculated if the power of signal of interest is known because the difference between $E_{b} / N_{0}$ and $I N R$ shows the power ratio of signal of interest to the self-interference $(S I R)$ when the noise power is fixed. $E_{b} / N_{0}$ and $I N R$ are defined as the power radio of signal of interest received to the thermal noise and self-interference signal to the thermal noise respectively.

As can be seen in Fig 3. (a), the self-interference signal received by the receiver is $20 \mathrm{~dB}$ higher than thermal noise, while the self-interference signal after AASIC is almost to noise level. Even in Fig 3. (b) (c), $I N R=40 d B$, $I N R=60 \mathrm{~dB}$, the self-interference signals after AASIC at the RF component are also extremely close to noise level. By comparison, the spectrum power of the self-interference signal $S 7$ received is much stronger than the residual selfinterference signal $S 3$ which is the interference signal after AASIC at the receiver RF component. Therefore we can conclude that the self-interference signal can be significantly reduced to almost noise level by using active analog selfinterference cancellation at the RF component in an ideal situation.

As shown in Fig 4., AASIC obtains $0 d B$ self-interference cancelation when $I N R=5 d B, 6 d B$ reduction when $I N R=$ $10 d B$ and $11.5 d B$ reduction when $I N R=15 d B$, while around $17 d B$ self-interference cancellation when $I N R=$ $20 \mathrm{~dB}, 37 \mathrm{~dB}$ reduction when $I N R=40 \mathrm{~dB}$ and $57 \mathrm{~dB}$ reduction when $I N R=60 d B$. Therefore, there are $3 \sim 5 d B$ residual self-interference occurs after the AASIC. This residual self-interference is mainly caused by the self-interference channel estimation error due to the thermal noise. A similar result can be found in [8]. Another remark is that about $3 d B$ residual self-interference occurs when $I N R \geq 20$, while more residual self-interference occurs when $I N R \leq 15 \mathrm{~dB}$. This is because the residual self-interference is closely related to the 


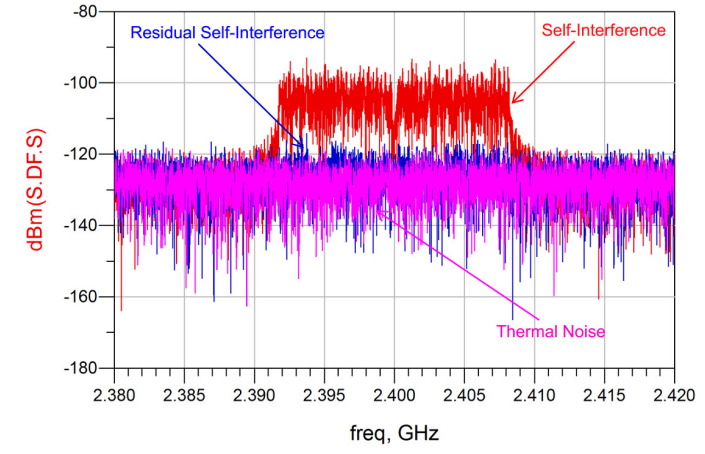

(a). INR $=20 \mathrm{~dB}$

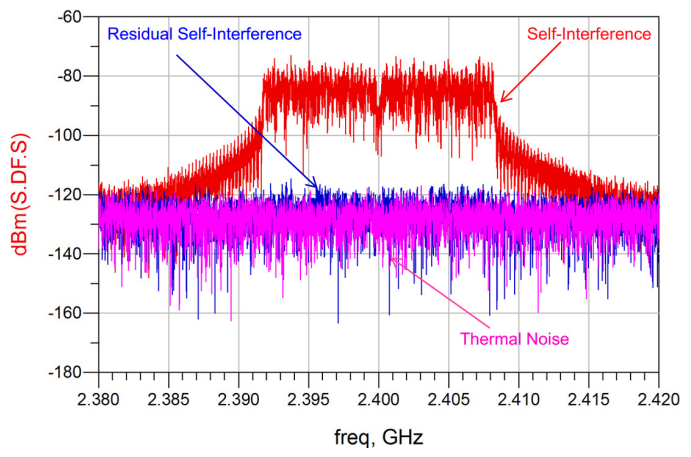

(b). INR $=40 \mathrm{~dB}$

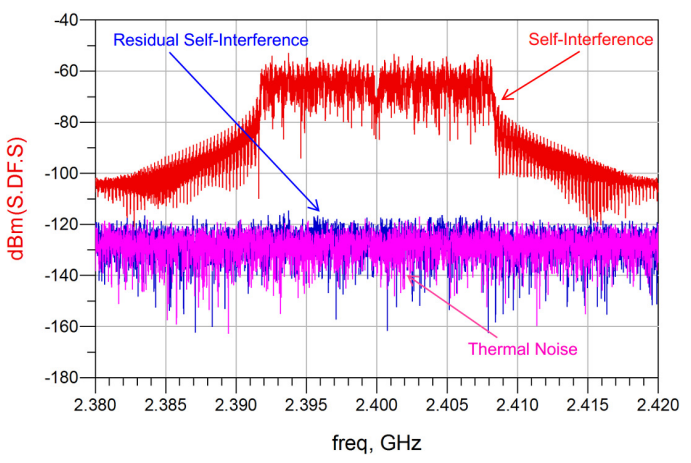

(c). INR $=60 \mathrm{~dB}$

Fig. 3. SpectrumPower of SI before and after AASIC for different INR

quality of subcarrier channel estimation. The higher the $I N R$ is, the more accurate the coefficients will be obtained. In the meantime, the less residual self-interference occurs. As what we thought, the best BER performance can be obtained only when there is no self-interference at the receiver chain and the BER performance degrads when residual self-interference is not zero due to fact that the self-interference signal can not be eliminated completely.

\section{CONCLUSION}

In this paper, a single channel $20 \mathrm{MHz}$ FuDu OFDM wireless system based on AASIC at the RF component is developed and elaborated and the AASIC is expressed in detail by mathematic model. Furthermore, we first study the BER performance of FuDu wireless via ADS-Matlab co-simulation.

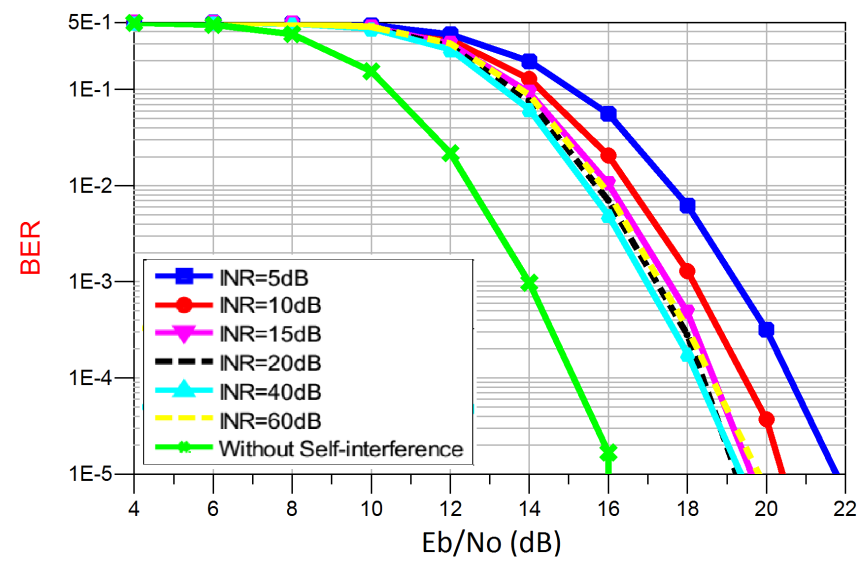

Fig. 4. The BER comparison of FuDu wireless for different INR

The simulation results show that the self-interference signal existing in wideband FuDu OFDM wireless can be significantly reduced to almost noise level. With respect to the analytical performance analysis, [9] gives the details.

In our design, RF radio is considered to be ideal. To design a more practical wideband FuDu OFDM system, the effect of $\mathrm{RF}$ impairment such as phase noise in the local oscillator, I/Q imbalance in RF modulation, frequency offset and nonlinearity of high power amplifier need to be taken into account in our following studies.

Compared to a single channel half-duplex wireless, FuDu wireless potentially doubles the throughput of wireless link. In order to further improve the efficiency of spectrum utility, we will combine FuDu OFDM radio with multimode MIMO radio to propose a new radio architecture: Full-Duplex Multimode MIMO-OFDM Radio.

\section{REFERENCES}

[1] A. Sahai, G. Patel, and A. Sabharwal, "Pushing the limits of full-duplex: Design and real-time implementation, http://arxiv.org/abs/1107.0607," in Rice University Technical Report TREE1104, June 2011.

[2] M. Duarte, C. Dick, and A. Sabharwal, "Experiment Driven Characterization of Full-Duplex Wireless Communications," IEEE Trans. Wireless Commun., vol.11, no. 12, pp4296-4307,Dec.2012.

[3] M. Jain, J. Choi, T. Kim, D. Bharadia, K. Srinivasan, P. Levis, S. Katti, P. Sinha, S. Seth, "Practical Real-Time Full Duplex Wireless," In ACM MOBICOM, 2011.

[4] P. Bahl, A. Adya, J. Padhye, A. Walman, "Reconsidering Wireless Systems With Multiple Radios,” In ACM SIGCOMM CCR, 2004.

[5] M. Duarte, A. Sabharwal, V. Aggarwal, R. Jana, K. Ramakrishnan, C. Rice, and N. Shankaranarayanan, "Design and Characterization of a Full-duplex Multi-antenna System for WiFi Networks," arXiv preprint arXiv:1210. 1639, 2012.

[6] Evan Everett, Achaleshwar Sahai, and Ashutosh Sabharwal, "Passive Self-Interference Suppression for Full-Duplex Infrastructure Nodes," arXiv preprint arXiv:1302. 2185, 2012.

[7] J. I. Choi, M. Jain, K. Srinivasan, P. Levis, and S. Katti, "Achieving Single Channel, Full Duplex Wireless Communications," in ACM MOBICOM, 2010.

[8] Achaleshwar Sahai, Gaurav Patel, Chris Dick and Ashutosh Sabharwal, "On the impact of Phase Noise on Active Cancellation in Wireless FullDuplex," arXiv preprint arXiv:1212.5462, 2012.

[9] Zhaowu Zhan, Guillaume Villemaud and Jean-Marie Gorce, "Analysis and Reduction of the Impact of Thermal Noise on the Full-Duplex OFDM Radio," submitted to IEEE Radio and Wireless Symposium 2014. 